\title{
Pituitary Magnetic Resonance Imaging in Pituitary apoplexy
}

Bellarbi. D, Azzoug. S, Rabehi. L, Terki. B, Chentli. F

Department of Endocrine and metabolic diseases, Bab El Oued Teaching Hospital, Algiers, Algeria

\section{INTRODUCTION}

Pituitary apoplexy is a rare endocrine emergency characterized by sudden onset of severe headaches, vomiting, visual abnormalities and pituitary dysfunction secondary to an acute hemorrhage or infarction within a pituitary adenoma.

\section{SUBJECTS AND METHODS}

Through a retrospective study from 2000 to 2014 about 23 cases of pituitary apoplexy were observed. Their mean age was 38.7 years +/- 10 years, with a male to female ratio $=2: 1$.

All patients benefited Magnetic resonance imaging (MRI).

\section{RESULTS}

Pituitary (MRI) showed macroadenomas in all cases. These macroadenomas invade the optical chiasma in $87 \%$, the cavernous sinuses in $48 \%$, the sphenoid sinus in $39 \%$. They have multidirectional extension in $22 \%$. Areas of intratumoral hemorrhage were evident on MRI in $94 \%$ leading to the positive diagnosis of pituitary apoplexy which confirms the importance of pituitary MRI when investigating pituitary apoplexy.

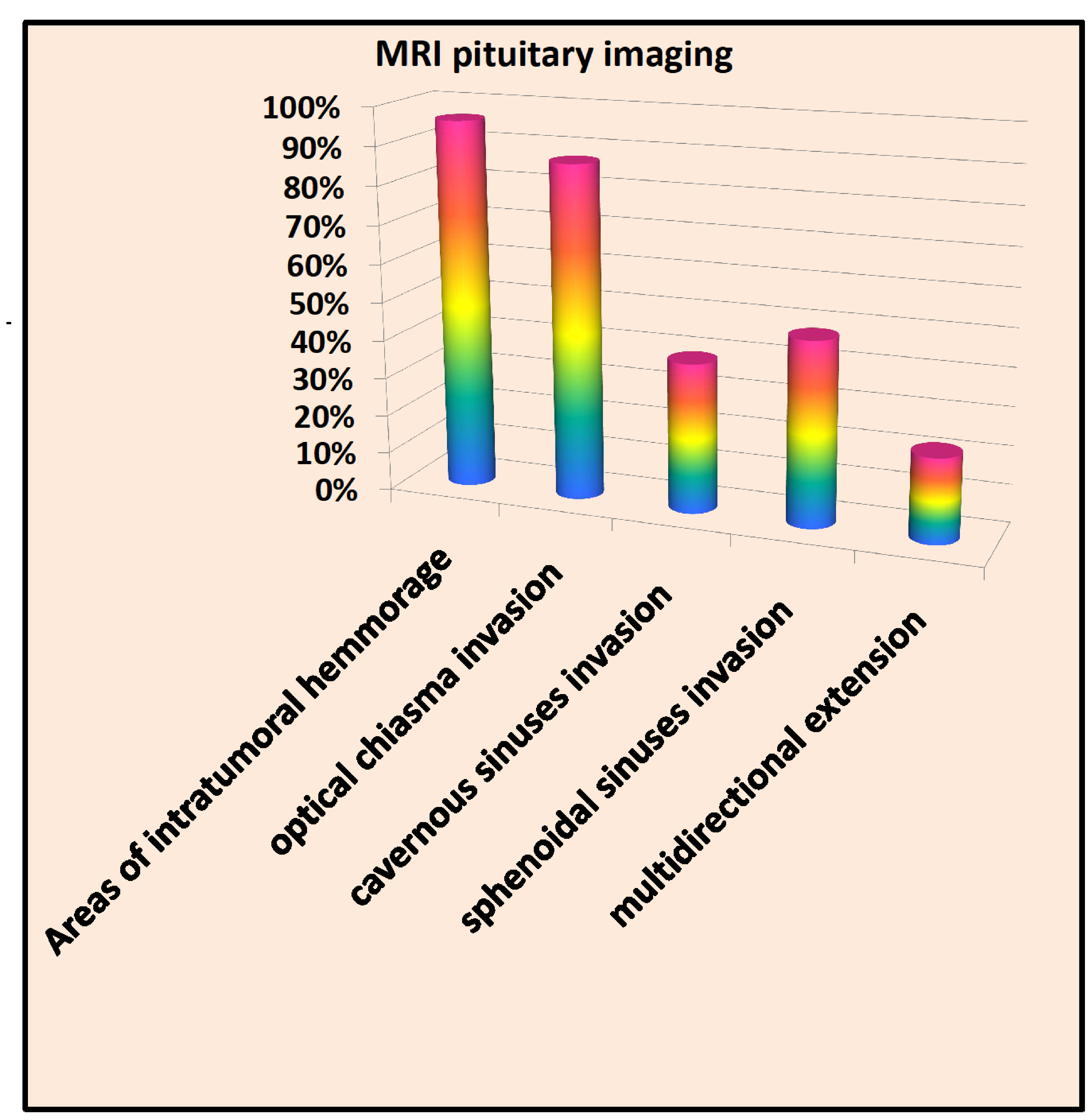

\section{CONCLUSION}

Pituitary apoplexy is a rare life-threatening clinical syndrome caused by infarction or hemorrhage within a pituitary adenoma. Magnetic resonance imaging (MRI) is the investigation of choice and has been shown to confirm the diagnosis in $94 \%$ of patients which is compatible with guidelines and results of the literature. Once pituitary apoplexy is diagnosed a multidisciplinary team approach is mandatory in order to improve the outcome of this condition. 